\title{
ECR discharge in a single solenoid field
}

\author{
R.A. Shaposhnikov, S.V. Golubev, I.V. Izotov, S.V. Razin, V.A. Skalyga
}

Institute of Applied Physics RAS, Nizhny Novgorod, Russian Federation, shaposhnikov-roma@mail.ru

Electron cyclotron resonance (ECR) ion sources nowadays are one of the widespread systems for ion beam production in basic and applied research. One of the main directions for its development is an increase of the extracted beam current, which can be realized by production of plasma with higher density. Significant growth of plasma density in an ECR discharge is possible only with an increase of microwave radiation frequency used for the heating. In modern ECR ion sources gyrotrons are increasingly used as a source of microwave radiation. Earlier at the IAP RAS it has been demonstrated that the use of $37.5 \mathrm{GHz}, 100 \mathrm{~kW}$ gyrotron radiation for plasma heating in a simple mirror magnetic trap allows to produce beams of light or multiply charged ions with record currents (up to $500 \mathrm{~mA}$ ) [1-3]. In these studies simple mirror traps were used for plasma confinement instead of conventional "minimum - B" system due to high complexity and production cost of the last ones in case of microwave heating frequency above $30 \mathrm{GHz}$. The key feature of mentioned research was possibility demonstration for plasma creation with density up to $2 \cdot 10^{13} \mathrm{~cm}^{-3}$ and electron temperature about $100 \mathrm{eV}$ under conditions of so-called quasigasdynamic regime of plasma confinement [4].

Such confinement regime is characterized by high density and so high electron Coulomb scattering into a loss cone that plasma losses from a trap are determined by its outgoing flux with ion sound velocity. It could be shown that in case of high enough plasma density its losses to a plasma chamber wall are always limited with ion sound velocity even without any magnetic confinement, plasma is confined mainly by electrostatic ambipolar potential. Under such conditions magnetic field of the trap is used for suppression of transversal plasma flux and gives additional geometrical factor in plasma lifetime. It is well known from fusion mirror machines studies that plasma lifetime in such systems is proportional to magnetic trap length and its mirror ratio (ratio between maximum and minimum B-field) [5].

However, the main disadvantage of a simple mirror trap is that it is favorable for development of magnetohydrodynamic instabilities, and additional methods are needed to suppress them. At the same time in IAP experiments magnetic traps with mirror ratio from 3 to 7 were used and such factor in plasma life time is not crucial. According to that it was proposed to investigate the prospects of creation a source of hydrogen ions on the basis of ECR discharge in a single solenoid sustained by powerful gyrotron radiation. Opposed to simple mirror trap this system is MHD stable and high power of microwave radiation could allow maintaining electron temperature on a sufficient level for high ionization efficiency of light gas.

The paper presents the first results on gas ECR breakdown studies in the single coil magnetic field and analysis of the prospects for the use of such plasma for ion beam production.

The first experiments were aimed to investigation of the possibility to realize the discharge in this system, determine plasma parameters and properties of the extracted ion beam. The experiments were carried out at SMIS 37 experimental facility where simple mirror magnetic trap was replaced with a single coil. Schematic view of the experimental setup (without gyrotron) is shown in fig. 1 . Pulsed gyrotron radiation with a frequency of $37.5 \mathrm{GHz}$, power up to $100 \mathrm{~kW}$ and duration up to $1.5 \mathrm{~ms}$ was used for plasma ignition and heating. The magnetic field in the coil varied from 1 to $4 \mathrm{~T}$ (ECR for $37.5=1.34 \mathrm{~T})$. The microwave radiation was injected into the plasma through a quasi-optical coupling system. Plasma was created in a specialized discharge chamber (working pressure from $10^{-7}$ to $10^{-3}$ Torr), the working gas (hydrogen) was fed into the discharge chamber along the system axis. Gas breakdown and plasma heating were performed under electron-cyclotron resonance conditions at the main harmonic. For beam extraction a two electrode extraction system was used. A plasma electrode with aperture diameter of $1 \mathrm{~mm}$ and a puller electrode with $3 \mathrm{~mm}$ aperture were placed in $6 \mathrm{~mm}$ from each other. Plasma electrode was placed outside of the coil in $12 \mathrm{~cm}$ from magnetic field maximum.

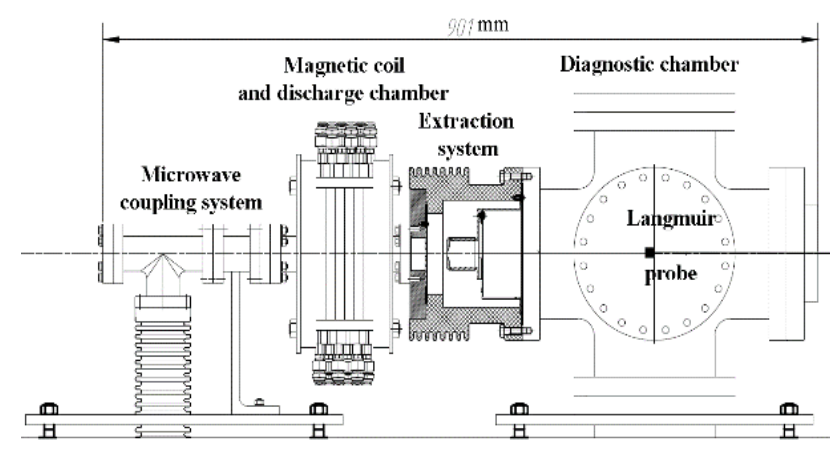

Fig. 1. Scheme of the experimental facility plasma part

At the first step experiments with a constant neutral gas injection were performed to study the possibility of the discharge ignition and to determine a threshold microwave power for it at various pressures. Breakdown curve plotted according to the experimental data is shown in fig. 2 .



Fig. 2. Breakdown threshold curve for hydrogen 
Also it was demonstrated that discharge could be realized only if maximum magnetic field in the chamber is above ECR value. It was clearly seen when the microwave pulse was shifted relatively magnetic field pulse from its maximum to the leading edge. It was done to observe breakdown behavior in case of rising in time magnetic field. The moment of discharge ignition was absolutely correlated with the time when magnetic field exceeded the ECR value.

All further investigations were performed in a pulsed mode of neutral gas injection into the system, which is standard for SMIS 37 operation. As the next step plasma parameters were studied using the regular Langmuir probe technique. The probe was placed in the diagnostic chamber on the system axis (see fig. 1) where magnetic field was weak enough (about $0.02 \mathrm{~T}$ ). The probe currentvoltage characteristics were recorded in a wide range of microwave power and gas pressure above the valve which was used for pulsed gas injection into the trap. Result of its processing for electron temperature determination is shown in fig. 3 .

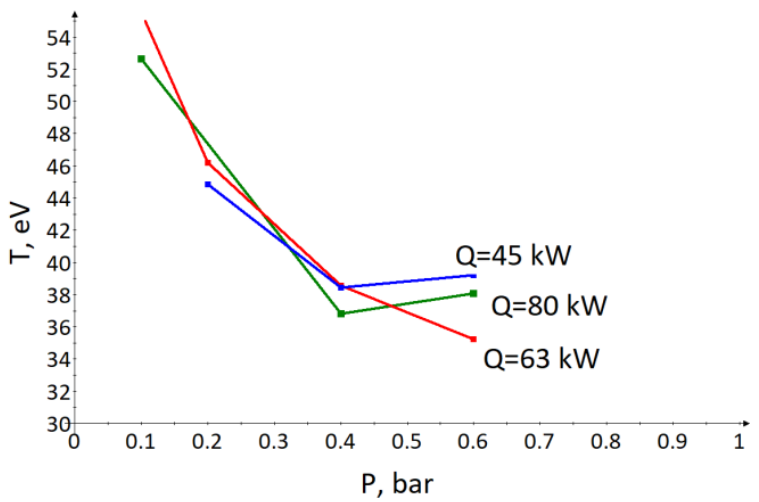

Fig. 3. Electron temperature dependence in hydrogen discharge for different microwave power and conditions of neutral gas injection

It is clearly seen that fine tuning of the gas pressure in the begging of microwave pulse by varying of the pressure above the valve and the moment of its opening could allow to obtain electron temperature up to $50 \mathrm{eV}$. Such temperature level is close to optimal for effective hydrogen ionization.

One of the main purposes of these experiments was to demonstrate a possibility of dense outgoing plasma flux production for high current ion beam formation. For these studies a two electrode extraction system was installed. Measured dependence of the extraction ion current on the voltage on extraction system is shown in fig. 4 in case of microwave power $\mathrm{Q}=80 \mathrm{~kW}$, gas pressure in the buffer chamber above the valve $\mathrm{P}=0.18$ bar.

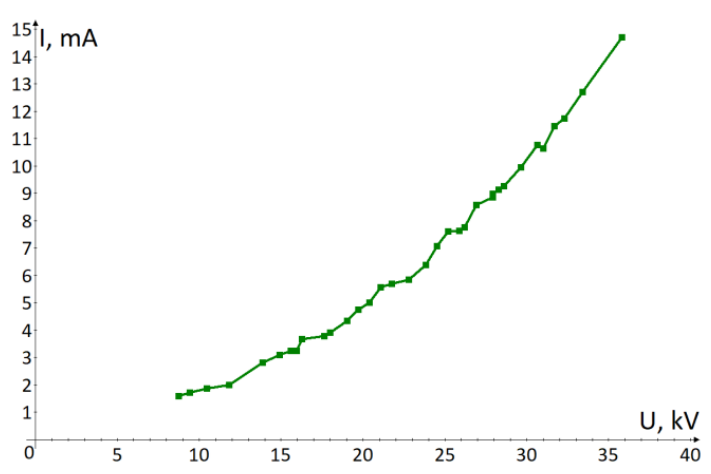

Fig. 4. Extracted ion beam current dependence on high voltage between electrodes

From figure 4 it can be seen that it was possible to obtain very high extraction current density values, $13 \mathrm{~mA}$ of total beam current corresponds to current density in the plasma electrode of more than $1.5 \mathrm{~A} / \mathrm{cm}^{2}$. It should be noted that the system parameters was not optimized, so, it might be possible to obtain better results. However, in experiments, which already have being carried out, high values of the ion current were demonstrated. This fact shows the prospects of the ion source design based on the system with one coil. High current ECR source of protons (or deuterons) with a single coil magnetic field looks interesting for applications where high ion current densities are required, such as ion beam injection to modern particle accelerators or compact powerful D-D neutron generators development.

\section{Acknowledgments}

Presented work was supported by the grant of Russian Science Foundation \# 16-19-10501.

\section{References}

1. S. Golubev, I. Izotov, S. Razin, A. Sidorov, V. Skalyga, A. Vodopyanov, V. Zorin, A. Bokhanov. High Current ECR Source of Multicharged Ion Beams. Nuclear Instruments and Methods in Physics Research B, v. 256, p. 537 - 542 (2007).

2. A. Sidorov, M. Dorf, A. Bokhanov, I. Izotov, S. Razin, V. Skalyga, V. Zorin, A. Balabaev, P Spädtke, J. Roßbach. Multiaperture ion beam extraction from gas-dynamic electron cyclotron resonance source of multicharged ions. Review of Scientific Instruments, 79, 02A317 (2008).

3. V. Skalyga, I. Izotov, S. Razin, A. Sidorov, S. Golubev, T. Kalvas, H. Koivisto, and O. Tarvainen. High current proton beams production at Simple Mirror Ion Source 37. Review of Scientific Instruments, v. 85 , no. 2, p. 02A702-1 - 02A7023 (2014).

4. V. Skalyga, I.Izotov, S.Golubev, A.Sidorov, S.Razin, A.Vodopyanov, O.Tarvainen, H.Koivisto, T.Kalvas. New progress of high current gasdynamic ion source. Review of Scientific Instruments. 87, 02A716 (2016)

5. V.V. Mirnov, D.D. Rutov, Pisma JTF, v.5, p. 678 (1979). 